\title{
Use of two-axis joystick for control of externally powered shoulder disarticulation prostheses
}

\author{
Robert D. Lipschutz, BSME, CP $;^{1 *}$ Blair Lock, MS; ${ }^{1}$ Jonathon Sensinger, PhD $;^{1-2}$ Aimee E. Schultz, MS; ${ }^{1}$ \\ Todd A. Kuiken, MD, PhD $^{1-2}$ \\ ${ }^{1}$ Center for Bionic Medicine, Rehabilitation Institute of Chicago, Chicago, IL; ${ }^{2}$ Department of Physical Medicine and \\ Rehabilitation, Feinberg School of Medicine, Northwestern University, Chicago, IL
}

\begin{abstract}
We explored a new method for simple and accurate control of shoulder movement for externally powered shoulder disarticulation prostheses with a two-axis joystick. We tested 10 subjects with intact shoulders and arms to determine the average amount of shoulder motion and force available to control an electronic input device. We then applied this information to two different input strategies to examine their effectiveness: (1) a traditional rocker potentiometer and a pair of force-sensing resistors and (2) a two-axis joystick. Three nondisabled subjects and two subjects with shoulder disarticulation amputations attempted to control an experimental externally powered shoulder using both control strategies. Two powered arms were tested, one with powered flexion/extension and humeral rotation and one with powered flexion/extension and adduction/abduction. Overwhelmingly, the subjects preferred the joystick control, because it was more intuitively linked with their shoulder movement. Additionally, two motions (one in each axis) could be controlled simultaneously. This pilot study provides valuable insight into an effective means of controlling high-level, externally powered prostheses with a two-axis joystick.
\end{abstract}

Key words: control strategies, externally powered prosthesis, force-sensing resistor, joystick, proportional control, proprioception, rocker potentiometer, shoulder disarticulation, shoulder excursion, upper-limb amputation.

\section{INTRODUCTION}

Fitting functional prostheses to individuals with shoulder disarticulation or humeral neck amputations is a major challenge. Cable-driven prostheses require precise fitting and harnessing to capture the required excursion from the user. Over $10 \mathrm{~cm}$ of excursion is required for the operation of a prosthesis, including $5 \mathrm{~cm}$ to open the terminal device and $>6 \mathrm{~cm}$ to flex the elbow. The most commonly used motions, scapular and biscapular protraction, typically generate $<10 \mathrm{~cm}$ of excursion. The required excursion of the prosthesis may be reduced to accommodate the limited excursion of the user by movement of the elbow flexion attachment or the inclusion of an excursion amplifier. A decrease in the required excursion comes with the associated cost of an increase in the force required to operate the prosthesis; many users find this force difficult to generate. The limited return in function and nonphysiological control are important factors in the high rejection rate of high-level prostheses [1].

\footnotetext{
Abbreviations: $\mathrm{DOF}=$ degree of freedom, $\mathrm{EMG}=$ electromyographic, FSR = force-sensing resistor, TMR = targeted muscle reinnervation.

*Address all corr espondence to Robert D. Lipschutz, BSME, CP; Center for Bionic Medicine, Rehabilitation Institute of Chicago, 345 E Superior St, Rm 1309, Chicago, IL 60611; 312-238-4137; fax: 312-238-2081.

Email: rlipschutz@northwestern.edu

DOI:10.1682/JRRD.2010.04.0072
} 
In an attempt to allow users with a shoulder disarticulation to control a prosthesis with less effort, prosthetists may use externally powered components or combinations of body-powered and externally powered components (hybrid prostheses). The use of externally powered components decreases the necessary excursion and force required of the user and may provide increased joint range of motion and/or grip strength. Commercially available powered components include terminal devices, elbows, and electronic wrist rotators. With the exception of one body-powered design, humeral rotators either are fixed or use passive friction turntables. Likewise, all commercially available shoulder joints are passive or lockable after manual positioning.

Externally powered components may be controlled with a variety of sensors, both digital and analog. Digital sensors include momentary contact switches and pull switches and will not be addressed in this article. Analog (proportional) sensors include force-sensing resistors (FSRs), motion-tracking sensors such as linear potentiometers or potentiometer-based joysticks, and electromyographic (EMG) sensors. Vodovnik and Rebersek found that motion-tracking sensors provide the best control of a single degree of freedom (DOF), followed by pressure sensors (such as FSRs), with EMG sensors ranking last [2]. At the transradial level, EMG sensors have the advantage of intuitively controlling the wrist and hand, but at the shoulder level, all sensors equally control the hand, wrist, or elbow unintuitively. Doubler and Childress have found that movement control (e.g., residual shoulder movement) provides good simultaneous control of two prosthetic DOFs [3-4] but have not compared their results with FSR or myoelectric control.

Subjects with externally powered shoulder disarticulation prostheses need to control multiple motors and would benefit substantially from control sources that allow precise, simultaneous control. Toward this end, we address clinically appropriate ways of using the residual shoulder as a control source. Specifically, we have looked at the conventional method of combining FSRs with a rocker potentiometer and the promising option of capturing shoulder motion with a two-axis joystick. Use of residual shoulder motion to control prosthetic components has been proposed for several decades. Several studies have used shoulder control on nondisabled subjects [3-5] or on subjects with an amputation controlling a remote prosthesis [6], and a few studies have looked at combined ipsilateral/contralateral shoulder control of an integrated prosthesis [7-10]. This article looks specifically at ipsilateral shoulder control sensors for use in a clinical setting, as well as the interfaces connecting the sensors to the subject.

\section{METHODS}

\section{Maximum Shoulder Force and Excursion}

An initial investigation looked at typical shoulder excursion (linear displacement) and force in nondisabled subjects. Two series of tests were performed on five men and five women. The first test used an oversized transparent socket with a telescoping rod/sheave apparatus that could be mounted superiorly, anteriorly, or posteriorly. The subjects were asked to relax as the rod was positioned in contact with their shoulder (one position at a time in each of the three locations). The subjects were then asked to move their shoulder complex through four different motions: elevation, depression, protraction, and retraction. Displacements during these four motions were measured as the change in translation of the telescoping rod at three locations (the superior position was used to measure both elevation and depression). Five trials were performed for each motion. Pressure measurements were also recorded from the same subjects, with a $0.786 \mathrm{~cm}^{2}$ point of contact. Data were collected for only three motions, because depression of the shoulder would pull away from the pressure gauge. Pressure data were then converted to force in Newtons.

\section{Shoulder-Control Techniques}

Three nondisabled subjects and two subjects with shoulder disarticulation amputations participated in the subsequent study. A certified prosthetist ${ }^{*}$ made custom bypass sockets for each nondisabled subject, consisting of clear thermoplastic sockets with an opening for their arm to exit. A large four-spoke mounting plate attached to the socket created a pass-through for the sound arm and permitted mounting of the prosthetic arm. Subjects with shoulder disarticulation amputations were fitted with conventional perimeter-frame-style sockets for mounting control inputs and electronic hardware. Subjects controlled a powered shoulder flexor and a powered humeral rotator. One subject with a shoulder disarticulation amputation

\footnotetext{
*American Board for Certification in Orthotics, Prosthetics \& Pedorthics.
} 
also used the joystick strategy to control shoulder adduction/abduction and shoulder flexion/extension. Both patients with shoulder disarticulation had undergone targeted muscle reinnervation (TMR) surgery [11-12] and used muscles in their chest to control elbow and hand movements simultaneously with shoulder movements. While the powered shoulder components used in this study are not commercially available, this control illustrates the potential control of commercially available devices such as elbows, wrists, and terminal devices.

Custom software, developed by the University of New Brunswick (New Brunswick, Canada), was used to provide real-time control of the prosthesis. The control paradigm used was proportional (velocity) control, which increases the speed of the motion as the magnitude of the command signal increases. It is the control paradigm used by Otto Bock (Minneapolis, Minnesota), Motion Control, Inc (Salt Lake City, Utah), and Liberating Technologies, Inc (Holliston, Massachusetts). Gains were individually tuned by a certified prosthetist for each sensor system. Two strategies for controlling two DOFs were tested: one using a traditional FSR/rocker potentiometer setup and the other using a two-axis joystick.

\section{Force-Sensing Resistor/Rocker Potentiometer Control}

The FSR/rocker potentiometer setup allowed for sequential control of humeral rotation and shoulder flexion/extension. For control of humeral rotation, two FSRs were placed on the lateral portion of the socket (Figure 1). One FSR was mounted anteriorly for operation of internal rotation and one FSR was mounted posteriorly for operation of external rotation. The velocity of humeral rotation was proportional to the force applied to the FSRs. This control strategy potentially could provide the subjects with cutaneous force feedback when they pressed on the FSRs. For control of shoulder flexion/ extension, a proportional rocker potentiometer was mounted above the shoulder on the superior aspect of the socket (Figure 1). This electromechanical component is a single-axis, center-zero rocking potentiometer (J3R rocker potentiometer single-axis joystick; ETI Systems, Carlsbad, California). The benefit of this device is that it can provide a proportional output, while traditional rocker "switches" used in externally powered prostheses allow only a single output level. The velocity of shoulder flexion/extension was proportional to the position of the rocker potentiometer.

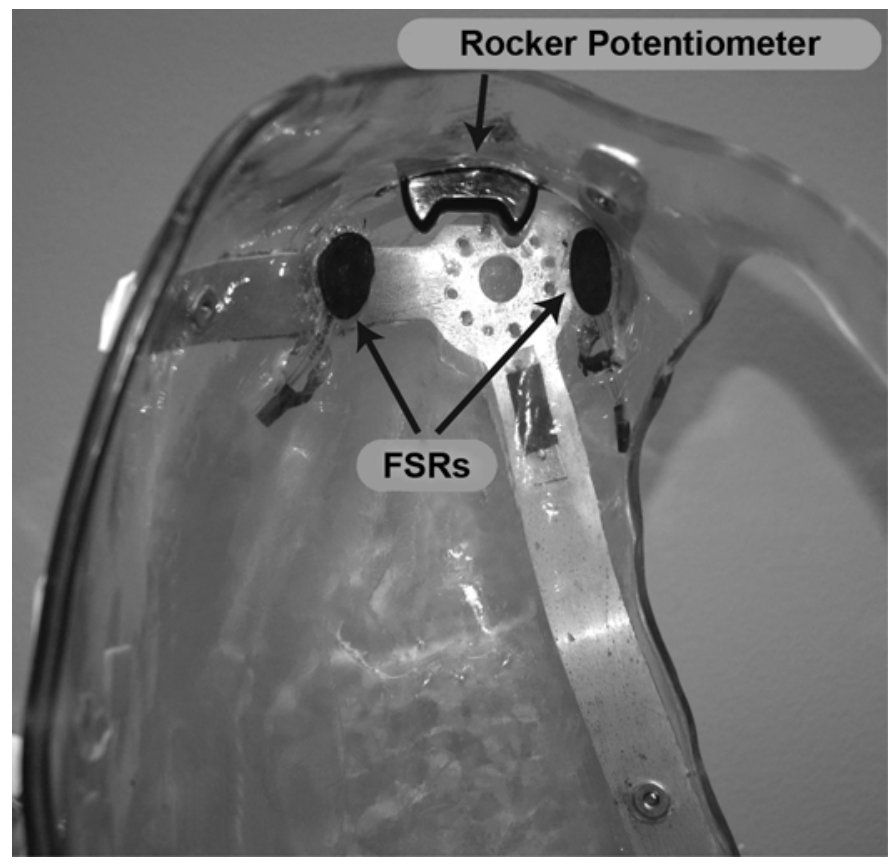

Figure 1.

Sagittal view of force-sensing resistors (FSRs)/rocker potentiometer setup in shoulder disarticulation prosthesis.

\section{Joystick Control}

A design with a two-axis joystick controlled two DOFs while enabling simple fabrication and calibration. The joystick consisted of two potentiometers aligned in orthogonal directions to measure shoulder displacements in two dimensions. Davalli and Sacchetti described the use of joysticks to control a powered hand, wrist, and elbow, although the linkage to the body was unclear [13]. In this study, the joystick provided input to motors that controlled anatomical shoulder movements. At first, the joystick was mounted medially on the socket on the distal portion of the shoulder segment (Figure 2). A shoulder cap, modified with an eyelet mounted superiorly to accept the extended rod of the joystick, was attached to the socket with three elastic-webbing straps. The design of the eyelet permitted the rod to slide and therefore prevented binding and inadvertent signals. The shoulder cap tracked shoulder movements and resulted in corresponding movements of the rod via the eyelet. When the joystick was used without the shoulder cap, the distal end of the rod was fed through a cylindrical sleeve. In one version of this design, the sleeve was bonded to a snap that attached it to a tightfitting shirt (Figure 3; also see Video 1, available online 


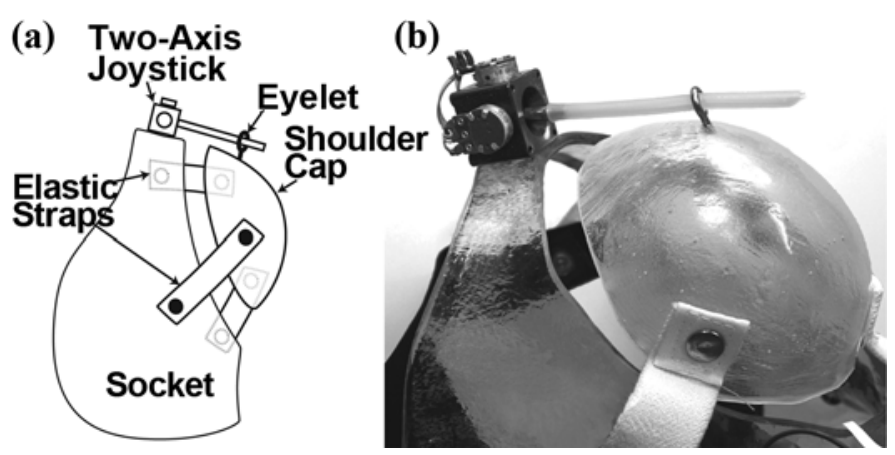

Figure 2.

(a) Diagram of setup for capturing shoulder movement using twoaxis joystick and shoulder cap. (b) Close-up of joystick and shoulder cap attached to prosthetic socket.
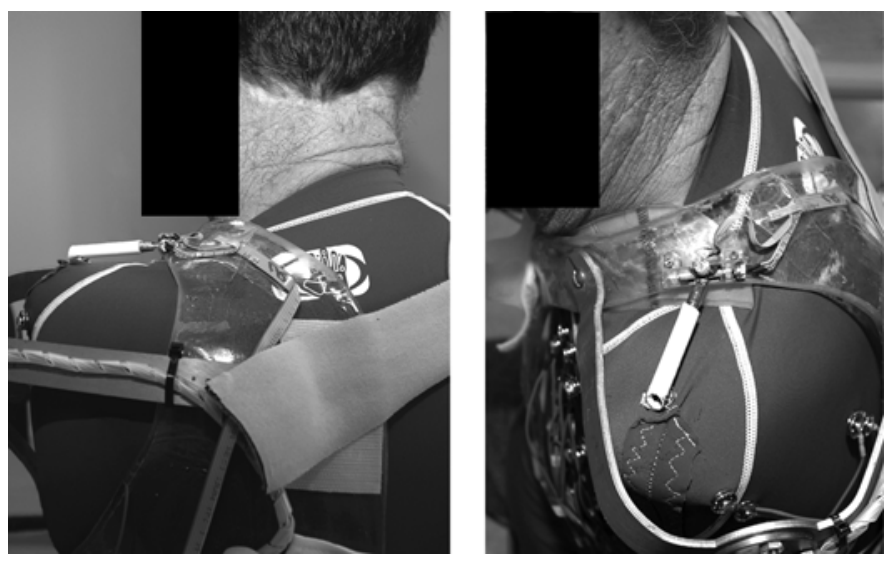

Figure 3.

Custom low-profile two-axis joystick with distal end of sheave affixed to spandex shirt. only). In the other version, the sleeve was bonded to a thin elastic strap secured to the socket (Figure 4; also see Video 2, available online only). Both of these designs used a hollow sheave, similar in size to a portion of a straw, to minimize the profile of the eyelet. A custom lowprofile two-axis joystick was used with the spandex shirt, and a commercially available miniature two-axis joystick (CTS 252, CTS Corporation; Elkhart, Indiana) was used with the elastic strap.

All five subjects tested a system in which shoulder elevation/depression controlled shoulder flexion/extension, and shoulder protraction/retraction controlled internal/external humeral rotation. One subject also tested a system in which internal/external humeral rotation was replaced by shoulder abduction/adduction.

\section{RESULTS}

\section{Maximum Shoulder Force and Excursion}

The maximum displacements and forces generated in the scapulothoracic joints of 10 nondisabled subjects are displayed in the Table. Significant differences were present between measured maximum displacements in elevation and depression, with substantial differences also present between maximum displacements in protraction and retraction. No significant differences existed between maximum forces generated via shoulder protraction, retraction, or elevation, or between male and female subjects for either excursion or force measurements.

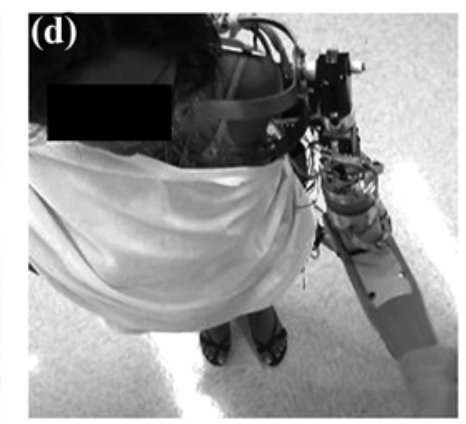

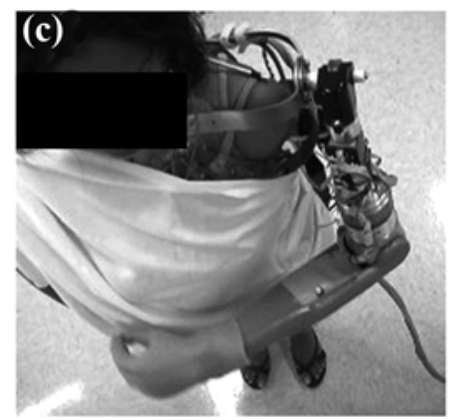

(a)

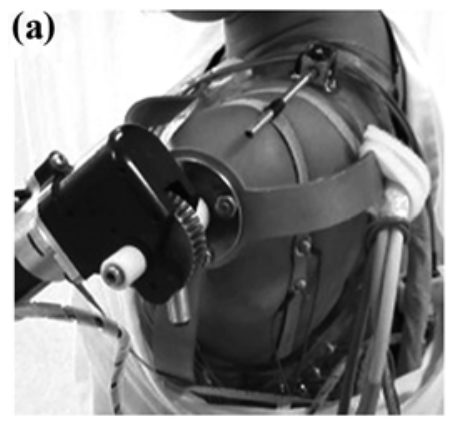

(b)

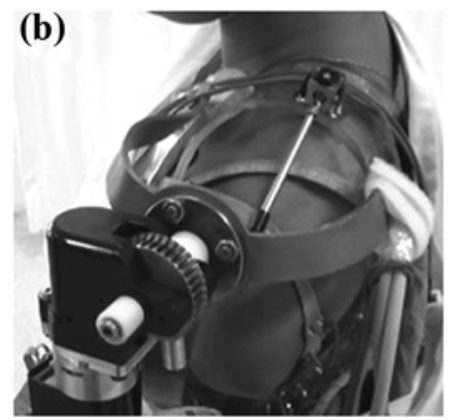

Figure 4.

Commercially available miniature two-axis joystick with distal end connected to elastic strap to capture shoulder movement and control powered shoulder and humeral rotator: (a) shoulder elevation produces shoulder flexion, (b) shoulder depression produces shoulder extension, (c) shoulder protraction produces internal humeral rotation, and (d) shoulder retraction produces external humeral rotation. 
Table.

Maximum shoulder displacements and forces of 10 nondisabled subjects.

\begin{tabular}{lcc}
\hline \multicolumn{1}{c}{ Movement } & $\begin{array}{c}\text { Displacement }(\mathbf{c m}) \\
\text { Average } \pm \text { SD }\end{array}$ & $\begin{array}{c}\text { Force (N) } \\
\text { Average } \pm \text { SD }\end{array}$ \\
\hline Protraction & $3.7 \pm 1.3$ & $21.5 \pm 6.2$ \\
Retraction & $2.8 \pm 0.9$ & $25.2 \pm 5.9$ \\
Elevation & $4.2 \pm 1.1$ & $25.7 \pm 6.2$ \\
Depression & $2.2 \pm 0.8$ & - \\
\hline SD = standard deviation. & \\
\hline
\end{tabular}

\section{Shoulder-Control Techniques}

Both nondisabled subjects and the subjects with a shoulder disarticulation were able to use the FSR/rocker potentiometer setup but had difficulty locating the appropriate sensor without occasionally hitting a different sensor. While they were all able to locate the sensors, the added delay of cautiously hunting for the appropriate sensor substantially inhibited their ability to control the prosthetic device. Although the subjects were able to feel the sensors, they could not appreciate significant cutaneous feedback from pressing on them.

For nondisabled subjects, control with the two-axis joystick was much better than that with the FSR/rocker potentiometer setup. Shoulder movements and humeral rotations were noticeably smoother and more fluid. Subjects also had fewer pauses in operation, because they were not required to search for the appropriate sensor; subjects reported that this made the control faster. They also reported that control with this strategy was easier and more intuitive and that it seemed more accurate.

\section{DISCUSSION}

Both strategies examined in this study were clinically viable for subjects with a shoulder disarticulation. Control with a joystick had clear advantages over control with the FSR/rocker potentiometer setup: it provided fast, accurate, and intuitive control in a large work space without the need to hunt for the sensors. It also provided simultaneous control of two DOFs.

The results of this study were somewhat surprising in that we expected the FSR/rocker potentiometer setup to be easier for the subjects to find and that it would provide the subjects with important cutaneous feedback that improved control. However, the joystick control system was clearly preferred by all our subjects, both nondis- abled subjects and those with shoulder disarticulations. The difference in performance of the two systems could be a result of the type and amount of feedback they provided to the patients. The joystick system provided the subjects with continuous control and proprioceptive feedback throughout their entire work space, whereas the FSR/rocker potentiometer setup only operated when touched (i.e., in a very small work space). The FSR/ rocker potentiometer setup clearly provided some cutaneous feedback when touched, while the joystick provided no cutaneous feedback because no resistance existed in the system. Thus, the superior proprioceptive feedback of the joystick appeared more beneficial than the limited cutaneous and proprioceptive feedback of the FSR/rocker potentiometer setup.

A spandex shirt or elastic strap provided substantially better joystick control than a shoulder cap in subjects with an amputation. Initially, joystick control did not work optimally for these subjects. The limited range of shoulder motion, coupled with the tendency of the shoulder cap to become displaced, prevented the subjects from having accurate control of the prosthesis. Custom shoulder caps did not capture shoulder movement with sufficient precision, because the elastic webbing held the cap in place too firmly, causing the shoulder to slip out of the cap if it moved too far. This effect was amplified when joint restrictions limited the subjects' movements. Spandex shirts and elastic straps provided better control because they more accurately captured the motion of the shoulder, which is an essential element in any motioncapture device.

Commercially available control software commonly uses proportional velocity control, and many research studies set the position, rather than the speed, of the prosthesis proportional to FSR strength or joint movement. Such a control paradigm is termed "proportional position control" or "servo control." Previous studies have shown that proportional position better controls the prosthetic joints than proportional velocity when shoulder motion is used as the input signal [3-4]. Because of software limitations, this study was only able to test proportional velocity control. The implications for sensor design still apply, however, and the use of proportional position control should only further highlight the benefits of using a two-axis joystick in clinical applications.

Several practical considerations contributed to the success of the two-axis joystick design. We initially placed the joystick on the medial side of the eyelet or 
sheath. In later designs, we moved the joystick to the lateral aspect of the socket. Lateral placement had two benefits: First, it shortened the length of the rod between the joystick and the sheath, which in turn amplified the rotational movement of the joystick. This amplification provided a greater range of joystick motion to be used, better controlling the prosthesis. Second, lateral placement allowed for integration of the joystick with the shoulder joint cosmesis, keeping an unobtrusive socket outline in the medial aspect, where it is more noticeable in collarless shirts.

One potential drawback of the joystick design was that it required a two-part socket, superior cutout, elastic strap, or spandex shirt. The need to provide two separate elements and have them joined by the joystick rod not only introduces some complexity to fabrication but also may be difficult for a person with shoulder disarticulation amputation to don and doff. In addition, parts and circuitry for the two-axis joystick are not currently commercially available for implementation on prosthetic devices.

Both of the subjects with shoulder disarticulation amputations tested in this study had undergone TMR [11-12] and thus had independent myoelectric control of elbow flexion/extension and terminal device open/close. We added the shoulder controller to provide inputs for externally powered shoulders and humeral rotators. These subjects both commented that shoulder control using the joystick provided higher fidelity control than their EMG sites. This being said, these subjects would opt to use both EMG and joystick control together to increase the number of simultaneously controlled prosthetic components, while they continue to prefer their TMR EMG sites to control the elbow and hand, because of the intuitive, physiologically appropriate portal they provide. Subjects who have not undergone TMR could still benefit from shoulder control using a two-axis joystick to control more distal joints such as terminal devices, wrists, and elbows.

These subjects with TMR used both EMG sensors and a motion-capture sensor. The FSR/rocker potentiometer setup did not work as well in parallel with TMR EMG. When a shoulder cap rigidly affixed to the main socket and FSR sensors was used, pressure applied against the socket to activate the FSRs frequently caused the EMG sensors to lose contact with the skin. A free-floating twoaxis joystick allowed for simultaneous control of the EMG sensors and the joystick, since shoulder movement did not come in contact with the socket and push away the EMG electrodes. This observation generally does not apply to persons with amputations who have not had TMR, because most patients are unlikely to use both EMG sensors and a motion-capture sensor.

\section{CONCLUSIONS}

Prosthetists can implement a two-axis joystick using commercially available components. The design takes less time to fabricate than proper placement of an FSR/ rocker potentiometer setup, which requires iterative adjustment to operate properly. As a result, prosthetists should consider using a joystick to improve control for subjects with a shoulder disarticulation. They must ensure that shoulder motion does not cause the entire socket to move and also pay close attention to providing minimal resistance to shoulder movement. This can be accomplished using a free-floating interface such as an elastic strap coupled to a freely moving potentiometer.

\section{ACKNOWLEDGMENTS}

\section{Author Contributions:}

Study concept and design: R. D. Lipschutz, B. Lock.

Acquisition of data: R. D. Lipschutz, B. Lock.

Analysis and interpretation of data: R. D. Lipschutz, B. Lock, J. Sensinger, A. E. Schultz.

Drafting of manuscript: R. D. Lipschutz, J. Sensinger, A. E. Schultz.

Critical revision of manuscript for important intellectual content: B. Lock, T. A. Kuiken.

Obtaining funding: T. A. Kuiken.

Study coordination and supervision: T. A. Kuiken.

Financial Disclosures: The authors have declared that no competing interests exist.

Funding/Support: This material was based on work supported by the DEKA Research and Development Corporation, Manchester, New Hampshire, contract W911NF-06-C-0001; Johns Hopkins University Applied Physics Laboratory, contract 908090; and National Institutes of Health/Eunice Kennedy Shriver National Institute of Child Health and Human Development, contracts R01 HD044798-01 and HHSN275200503402C/N01-HD-5-3402.

Additional Contributions: The authors thank Joseph E. Barton, $\mathrm{PhD}$, for his participation in the planning and data collection phases of the preliminary shoulder excursion and force experiments.

Institutional Review: All tests were performed with written, informed consent and institutional review board approval.

Participant Follow-Up: The authors plan to inform participants of the publication of this study. 


\section{REFERENCES}

1. Biddiss EA, Chau TT. Upper limb prosthesis use and abandonment: A survey of the last 25 years. Prosthet Orthot Int. 2007;31(3):236-57. [PMID: 17979010]

DOI:10.1080/03093640600994581

2. Vodovnik L, Rebersek S. Information content of myocontrol signals for orthotic and prosthetic systems. Arch Phys Med Rehabil. 1974;55(2):52-56. [PMID: 4272626]

3. Doubler JA, Childress DS. Design and evaluation of a prosthesis control system based on the concept of extended physiological proprioception. J Rehabil Res Dev. 1984;21(1):19-31. [PMID: 6527287]

4. Doubler J, Childress DS. An analysis of extended physiological proprioception as a prosthesis-control technique. J Rehabil Res Dev. 1984;21(1):5-18. [PMID: 6527290]

5. Durfee WK, Mariano TR, Zahradnik JL. Simulator for evaluating shoulder motion as a command source for FES grasp restoration systems. Arch Phys Med Rehabil. 1991; 72(13):1088-94. [PMID: 1741661]

6. Kyberd PJ, Poulton A, Sandsjo L, Jonsson S, Jones B, Gow D. The ToMPAW modular prosthesis: A platform for research in upper-limb prosthetics. J Prosthet Orthot. 2007; 19(1):15-21. DOI:10.1097/JPO.0b013e31802d46f8

7. Simpson DC. Externally powered prosthesis for complete arm replacement. Phys Med Biol. 1972;17(1):110.

8. Simpson DC, Kenworth G. Design of a complete arm prosthesis. Biomed Eng. 1973;8(2):56-59. [PMID: 4691519$]$

9. Simpson DC, Smith JG. An externally powered controlled complete arm prosthesis. J Med Eng Technol. 1977;1(5): 275-77. [PMID: 597551] DOI:10.3109/03091907709162194

10. Simpson DC. The choice of control system for the multimovement prosthesis: Extended physiological proprioception (EPP). In: Herberts P, Kadefors R, Magnusson R,
Petersen I, editors. The control of upper-extremity prostheses and orthoses. Springfield (IL): Thomas; 1974. p. 146-50.

11. Kuiken TA, Miller LA, Lipschutz RD, Lock BA, Stubblefield K, Marasco PD, Zhou P, Dumanian GA. Targeted reinnervation for enhanced prosthetic arm function in a woman with a proximal amputation: A case study. Lancet. 2007;369(9559):371-80. [PMID: 17276777]

DOI:10.1016/S0140-6736(07)60193-7

12. Kuiken TA, Dumanian GA, Lipschutz RD, Miller LA, Stubblefield KA. The use of targeted muscle reinnervation for improved myoelectric prosthesis control in a bilateral shoulder disarticulation amputee. Prosthet Orthot Int. 2004; 28(3):245-53. [PMID: 15658637]

13. Davalli A, Sacchetti R. Mini joystick for upper limbs prostheses. Proceedings of the 2002 Myoelectric Controls Symposium; 2002 Aug 21-23; Fredericton, Canada. Fredericton, Canada: Institute of Biomedical Engineering, University of New Brunswick; 2002 Aug 21-23. p. 86-89.

Submitted for publication April 22, 2010. Accepted in revised form September 10, 2010.

This article and any supplementary material should be cited as follows:

Lipschutz RD, Lock B, Sensinger J, Schultz AE, Kuiken TA. Use of two-axis joystick for control of externally powered shoulder disarticulation prostheses. J Rehabil Res Dev. 2011;48(6):661-68.

DOI:10.1682/JRRD.2010.04.0072 
